\title{
Ignaz von Olfers y los estudios lingüísticos americanos de Wilhelm von Humboldt
}

\author{
Ignaz von Olfers and Wilhelm von Humboldt's \\ linguistic studies on the American native languages
}

Manfred Ringmacher ${ }^{1}$

\begin{abstract}
Resumen
Entre los múltiples trabajos que llevó a cabo el intelectual prusiano Wilhelm von Humboldt, los estudios de las lenguas del mundo ocupan un lugar de destaque. Además de su predilección por el griego antiguo y de sus bien conocidas investigaciones sobre vasco, en la península ibérica, y sobre el kawi, en la isla de Jawa, este estudioso se dedicó con particular ahínco a analizar las lenguas indígenas del continente americano. Considerando que Wilhelm, a diferencia de su hermano Alexander, nunca estuvo en América, sus fuentes para esos trabajos son siempre de segunda mano. Uno de sus informantes más inteligentes y confiables fue Ignaz von Olfers, un naturalista también prusiano, que estuvo en Brasil en dos ocasiones, primero como integrante de la legación diplomática prusiana (1817-1821) y después como encargado de negocios (1826-1828). Mediante diversas fuentes documentales, en particular la correspondencia entre los dos personajes, este artículo muestra cuál fue la contribución de Ignaz von Olfers para los estudios lingüísticos de Wilhelm von Humboldt.
\end{abstract}

Palabras llave: lenguas americanas, lingüistas prusianos, indios de Brasil, Wilhelm von Humboldt, Ignaz von Olfers.

\begin{abstract}
Of particular interest among the numerous works by the Prussian intellectual Wilhelm von Humboldt are his studies on the languages of the world. In addition to his fondness for ancient Greek and his well-known research on the Basque language, in the Iberian Peninsula, and on the Kawi language on the island of Jawa, he devoted himself passionately to the analysis of the native languages of the American continent. As Wilhelm - unlike his brother Alexander - never travelled to America, the sources of his works were all secondary. One of his most intelligent and reliable informants was Ignaz von Olfers, also a Prussian naturalist, who stayed in Brazil twice, first as member of the diplomatic mission of Prussia (1817-1821) and later as Chargé d'Affaires (1826-1828). Through various documentary
\end{abstract}

${ }^{1}$ Edición de las obras lingüísticas de Wilhelm von Humboldt en la Academia de la Ciencias de Berlín-Brandenburgo - Jägerstrasse 22-23, Berlín 10117; e-mail: ringmacher@bbaw.de. 
sources, especially the correspondence between these two figures, this article shows the contribution of Ignaz von Olfers to the linguistic studies of Wilhelm von Humboldt.

Key words: American languages, Prussian languages, indigenous peoples of Brazil, Wilhelm von Humboldt, Ignaz von Olfers.

\section{Coleccionar lenguas y pensar la lengua}

El primer cuarto del siglo XIX, que vio la emancipación de América del Sur de los viejos poderes coloniales, España y Portugal, se presenta en una perspectiva europea transpirenaica como una época de las aperturas sudamericanas. Los poderes coloniales habían sido muy cautos con la admisión de observadores extranjeros a sus posesiones americanas, mientras los nuevos estados independientes tuvieron un interés vital en cultivar contactos regulares y profundos con el resto del mundo.

El interés de los naturalistas europeos - franceses, austríacos, bávaros, prusianos - por las riquezas naturales de Brasil fue grande: "En ciertos aspectos el Brasil es para el naturalista un mundo nuevo" (Eschwege 1818, parte I, p. VII). Llegaron y coleccionaron datos zoológicos, botánicos, mineralógicos y también etnográficos, todo lo que cabía en un marco de referencia geográfico. Observando a los hombres, habían descubierto la importancia no solamente de la lengua en general, sino de las diferentes lenguas. La documentación de las lenguas indígenas en listas de palabras empezó, sin duda, por el interés por los nombres indígenas de las riquezas naturales encontradas; pero aun las listas más elementales solían incluir otros vocablos, tales como nombres de parentesco o de partes del cuerpo. La lingüística de la época no era capaz de corregir este desequilibrio e insistir en la importancia de los verbos y de los textos enteros, porque los lingüistas se interesaban muy poco por la diferencia de las lenguas y mucho más en la relación entre la lengua (cada lengua) y el pensamiento.

Wilhelm von Humboldt tiene una posición de excepción en su época, precisamente porque enfoca la lengua a la vez como pensador del lenguaje humano (en su unidad y diferenciación) y coleccionador de lenguas. En 1799 hizo un viaje a España, en compañía de su familia; ahí descubrió a los vascos y su lengua y volvió al país vasco en 1801 para encontrar a cultores vascos de la lengua vasca. ${ }^{2}$ Ocupándose del vascuence descubrió las lenguas americanas, ${ }^{3}$ esta vez sin viajar. Las conoció a través de las gramáticas y diccionarios

\footnotetext{
${ }^{2}$ En la edición crítica de las obras lingüísticas de Wilhelm von Humboldt (cf. Ringmacher 2012:28), los textos ligados a la temática vasca son el objeto de una sección aparte. Cf. Humboldt (2010, 2012).

${ }^{3}$ Los textos de Wilhelm von Humboldt sobre lenguas americanas son el objeto de otra sección de la edición crítica de las obras lingüísticas de Wilhelm von Humboldt. Cf. Humboldt (1994, 2009, 2011, 2013, en prensa).
} 
compuestos por los misioneros católicos (españoles y portugueses) en el Sur y protestantes en el Norte (Estados Unidos). Coleccionar lenguas significaba para él, sobre todo, coleccionar libros (cf. Mueller-Vollmer 1994; Ringmacher 2012:20-21). Si esta ecuación no tiene un valor absoluto, el mérito es de Ignaz von Olfers, un naturalista que para poder viajar al Brasil ingresó en el servicio diplomático prusiano. Él apoyó con gran eficacia la búsqueda de libros sobre lenguas americanas de Wilhelm von Humboldt, y además aprovechó sus contactos con naturalistas que trabajaban con indios para procurar observaciones inéditas sobre las lenguas.

\section{"Llegado a un punto interesante": Ignaz von Olfers}

Ignaz von Olfers nació en 1793 en la ciudad de Münster en Westfalia. Su padre era jurista del príncipe-obispo de Münster. Desde 1798 fue socio y, más tarde, único dueño de un banco. En 1803, al término del principado de los obispos, se le confirió la nobleza hereditaria. El hijo Ignaz frecuentó el liceo de su ciudad y de 1812-15 pasó el trienio académico en la universidad de Göttingen realizando estudios de ciencias naturales, que concluyó con un doctorado en medicina (Rave 1962:108). Recomendado por el zoólogo berlinés Lichtenstein, fue incluido en la misión diplomática prusiana ${ }^{4}$ que en otoño de 1817 (Hermannstädter 2002:35) viajó a Rio de Janeiro, donde se había instalado la corte del rey de Portugal; ahí permanecería hasta el 26 de abril de 1821 (Schwarcz 2002:480 y 484).

Lichtenstein esperaba poder enriquecer las colecciones de los museos de ciencias naturales de su país con la ayuda de Olfers. En junio de 1818, Olfers encontró al botánico Friedrich Sellow, quien ya desde 1814 estaba en Brasil, reuniendo colecciones de objetos naturales. En agosto de 1818, ambos viajaron para Minas Gerais y al interior de la provincia de São Paulo. Entre septiembre de 1818 y abril de 1819 estuvieron en Villa Rica (Ouro Preto), explorando los alrededores. En junio de 1819 pasaron por la planta siderúrgica de Ipanema, cerca de Sorocaba, un puesto al que llegaban regularmente informaciones de la capital. Olfers fue llamado a Rio de Janeiro por asuntos de negocios y Sellow continuó el viaje en solitario. El proyecto de Olfers de reanudar el viaje interrumpido no se materializó, y en 1821 el cuerpo diplomático acompañó al rey en su retorno a Lisboa (Hermannstädter 2002:32-37).

\footnotetext{
${ }^{4}$ Esta misión comprendía a: "Conde Flemming, enviado extraordinario" y "v. Allfers, secretario de legación" (Hassel 1816:495). En Rio de Janeiro se hablaba de los diplomáticos y el naturalista Sellow: "un Prussien, le comte de Flemming, ayant demandé l'autorisation de visiter Minas avec un secrétaire et un botaniste, la dite autorisation lui fut concédée, mais à lui seul, individuellement, à l'exclusion de son secrétaire, ce qui fit qu'il renonça à sa mission" (Lima-Barbosa 1923 :260). No obstante, Sellow y Olfers viajaron a Minas Gerais en 1818; el rumor negativo puede referirse solamente a una falta inicial de éxito al solicitar un permiso.
} 


\section{El contacto con los hermanos Humboldt}

Olfers había establecido contacto con Alexander von Humboldt ya en 1816 en Paris, precisamente cuando se embarcaba para Brasil (Rave 1962:112). La relación con Wilhelm parece datar de 1821, el año del retorno de Olfers al Portugal europeo. La serie de cartas conservadas se inicia con una carta de Wilhelm von Humboldt del 27 de junio de 1821, en la que responde a una carta - no conservada - de Olfers del 24 de marzo. Humboldt está en su propiedad silesiana de Ottmachau (hoy día Otmuchów en Polonia) y ha dispuesto que le sean encaminados solamente los envíos postales sencillos, sin anexos eventualmente voluminosos. De tal manera, Humboldt lee únicamente la carta, sin los anexos lingüísticos mencionados por Olfers, unas pocas hojas de papel, pero quizás con un formato algo más grande que la propia carta.

Dos documentos que se encuentran entre los papeles lingüísticos de Wilhelm von Humboldt, que hoy se conservan en la Preussische Staatsbibliothek de Berlín y en la Biblioteca Jaguelónica de Cracovia (Kraków) ${ }^{5}$, pueden corresponder a lo que Olfers debe haber enviado el 24 de marzo de 1821.

\section{El primer documento}

En el primero de los cuatro tomos de las "Listas de palabras de lenguas americanas" (Wortverzeichnisse amerikanischer Sprachen, Coll. ling. fol. 4548, vol. 1, fol. 68-73) de la Staatsbibliothek (y antiguamente de la biblioteca lingüística de Humboldt) uno de los títulos dice "Botocudos" (Mueller-Vollmer 1993:193). Eduard Buschmann, el colaborador de Humboldt, escribió: "Lista de palabras de los Botocudos" (Wortverzeichniß der Botocudos, Coll. ling. fol. 45 , fol. 68 r) y el propio Humboldt anotó en un folio aparte:

Botocudos.

Comunicación del señor v. Olfers.

Las palabras de la primera columna provienen del capitán de cabalería Marlière, anotadas en el Rio Doce, de la boca de un muchacho capturado. La segunda es del señor Sellow, quien ha vivido mucho tiempo entre los Botocudos no lejos de la costa del Rio Belmonte (fol. $69 \mathrm{r})$.

La lista propiamente tal fue escrita por Olfers; ${ }^{6}$ empieza con una serie de nombres de parentesco y nombres de partes del cuerpo:

\footnotetext{
${ }^{5}$ Cf. la descripción de la parte manuscrita del legado lingüístico de Wilhelm von Humboldt en Mueller-Vollmer (1993). Un apéndice (Mueller-Vollmer 1993:407-444) se refiere a la colección de libros impresos de lenguas del mundo (no solamente las americanas).

${ }^{6}$ Identificación por Ulrich Moritz de la Historische Arbeitsstelle del Naturkundemuseum, Berlín.
} 
según Marlière

Tà.

Tijupeé.

Anaclem.

Hijo.

Hija.

Hermano.

Hermana.

Tío.

Abuelo.

Cabeza.

Cabellera.
Anaclem.

Jekjáme.

Jakuete.

Emm.

Apú.

Manháca.
Kléne. según Sellow

Jikanne.

Jipue.

Juprake.

Jukutéa.

Koaincai. (fol. 70 r)

En 7 páginas (fol. 70r-73r) contiene 174 entradas, sobre todo sustantivos del ambiente natural y social, pero después de "Flecha", también el verbo "Matar" (fol. 70v), o después de una serie de nombres de pájaros, también los verbos "Comer" y "Beber" (fol. 71v). A partir del fol. 72r aparecen unas entradas que (por lo menos en alemán) son adjetivos ("muy grande", "duro, fuerte", “alto", "dulce", "sabroso", "hermoso", "feo", “enfermo", "pequeño", "fuerte”, "abundante") y en la última página algunos imperativos ("Ven aca!", "Dame la mano!", “Que nos vemos!”, “Toma!’, “Abrázame!”). La última entrada (con la traducción solamente de Sellow, ${ }^{7}$ no de Marlière): "No sé. Gajé nuke" (fol. 73r).

\section{El segundo documento}

Entre los papeles de Humboldt está también una "Lista de algunas palabras de las lenguas de las tribus indígenas: Coroádos, Puris, Córópós, Panarás, Chicriabás, con sus sinónimos según la llamada Lingua geral", tres folios en formato oblongo sin paginación. Sin duda es un accidente que hoy esta lista esté en un mismo tomo (Coll. ling. fol. 150) con la gramática que Humboldt compuso del Onondago, una lengua de América del Norte (cf. Humboldt 2013:257-340). La mano autora del manuscrito es desconocida. Esta lista comprende 155 entradas, que no se indican en todas las seis lenguas comparadas. Aquí también, como en la primera lista, la mayor parte del material es de sustantivos con significados concretos, pero a partir del fol. $* 2 \mathrm{v}$ aparecen también nombres de colores ("negro", “el negro", "blanco", “el blanco", "rojo", "verde”, “azul”),

\footnotetext{
7 "Jagi nuke ich weis nicht" (SI Sellow, Tagebücher, Nr. 71., fol. 5r). La fidelidad de la copia hecha por Olfers es siempre problemática. En principio, las notas tomadas por Sellow pueden ser leídas solamente por quien sabe la lengua anotada.

${ }^{8}$ Los nombres de los colores son adjetivos en la lengua europea; según se nota en la
} 
los pronombres personales, los numerales hasta 5 , varias parejas de contrastes adjetivales, imperativos y finalmente cinco frases brevísimas ("Esto no es bueno", "He comido", "Esto es hermoso", "Adónde vas?", "De dónde vienes?", fol. *3 r).

Mueller-Vollmer (1993:336) se pregunta por qué estas seis listas paralelas no han sido incluidas en las "Listas de palabras de lenguas americanas" (Coll. ling. fol. 45-48), cada una en su lugar. La respuesta es que, de las seis lenguas comparadas, sólo dos, el Coroado y la Língua Geral (una variedad tardía del tupí) $)^{9}$, ya eran suficientemente conocidas. De las otras dos, Coropó y Purí, se tenía conocimiento a través de una lista comparativa publicada por Eschwege (1818, entrega 1:165-171) ${ }^{10}$, que eran muy semejantes al Coroado. ${ }^{11}$ Los grupos Panará $^{12}$ (Kayapó) y Xikriabá, ${ }^{13}$ o sea, el material de la cuarta y quinta columna de esta lista, estaban considerablemente menos bien documentados.

Ehrenreich (1894:115) conoce para la lengua Kayapó, antes de Martius (1867 (2):134-135), solamente dos listas: la de Pohl (1832-37 (1):447-448), que constituye la base de la lista de palabras Kayapó de Martius, y la lista de Saint-Hilaire (1847 (2):108-109), que proporcionó algunas palabras más a Martius. Pohl habla de Cayapós, Saint-Hilaire de Coyapós; ambos ignoran el etnónimo Panará, aunque Saint-Hilaire ha oído que entre los Kayapó salvajes el nombre que se dan a sí mismos sería Panariá (p. 106). Sin embargo el biógrafo

columna tupí ("língua geral"), las dos formas que en alemán se presentan como sustantivos por el artículo no designan el color de los objetos, sino las dos razas humanas del Brasil no indígena. Tal debe haber sido la expectativa del que preguntaba; los informantes, a lo que parece, no la entendían.

${ }^{9}$ El vocablo indicador nitio mbae 'nada' de la sexta columna (Coll. ling. fol. 150, fol. *2 v) prueba que no se trata del tupinambá, lengua tribal, sino de una forma antigua de la lengua de comunicación interétnica (Edelweiss 1969:282), de acceso exclusivamente escrito en aquel entonces (Anónimo 1795:53; la construcción completa habría sido: "Não he nada, Nitio mbae oico", p. 54).

${ }^{10}$ Existe una lista, igualmente de los idiomas Coroado, Coropó y Purí, de Freyreiss (1968:7377), impresa - sin duda con muchos errores - solamente en el siglo XX. La coincidencia parcial de las listas se explicaría si las listas fuesen derivadas de notas de campo más extendidas.

${ }^{11}$ Cf. la familia de lenguas purí de Rodrigues (1999:167), extinta entretanto, entre las lenguas Macro-Jê.

${ }^{12}$ Panará o Panariá es otro nombre de los Kayapó (del Sur), uno de los grupos de la familia lingüística Jê (Rodrigues 1999:167). En el material Kayapó publicado por Saint-Hilaire se halla la ecuación: "Un Indien, panaria" (Saint-Hilaire 1847 (2):108; cf. Rodrigues, Dourado 1993), un intento de anotar la auto-designación panariá.

${ }^{13}$ El etnónimo Xikriabá o Xakriabá de un grupo que hablaba una lengua jê (cf. Rodrigues 1999:167) se explica por el vocablo kayapó xikriá ,mano’ de Saint-Hilaire. Este nombre parece referirse a un vendaje característico en la muñeca para protegerla de la cuerda del arco después del tiro (Martius 1867 (1):278). 
de Sellow aún vio entre los papeles de éste último vocabularios de los "Paranas" o "Cojopó" (Urban 1893:193), que entretanto se extraviaron o perecieron en la Segunda Guerra Mundial.

En cuanto al Xikriabá (o Xakriabá), Eschwege (1830 (1):95-96) publicó una lista de "palabras de la lengua de los Xigriabás". En su diario registra en octubre de 1816 que estas palabras se las dictó una mujer solamente después de muchos ruegos, "porque en la presencia de extraños se recatan de hablar su lengua" (S. 93). De nuevo, la coincidencia parcial puede tener su origen en el empleo de notas de campo. No existen pruebas positivas de la participación de Olfers en la elaboración de la lista de seis lenguas (Coll. ling. fol. 150), pero tampoco existen pruebas negativas. Es bastante probable que Olfers haya sido quien tuvo acceso a las notas de campo de otros y que las empleó de igual manera como en el caso de la "língua geral", donde el material fue tomado del Diccionario anónimo (Anónimo 1795).

\section{El juicio de Humboldt}

En esa época, por tanto, la documentación de las lenguas amerindias vivas de Brasil fue una tarea de los naturalistas, no obstante su especialidad estuviese centrada en otros dominios. En el mejor de los $\operatorname{casos}^{14}$, ellos estaban conscientes de hallarse lejos de ofrecer una descripción suficiente: "Estes glossarios não offerecem o conhecimento subtil e satisfactorio do caracter grammatical das linguagens, mas sim sômente um aspecto superficial dos primeiros elementos, que as compoem" (Martius 1867 (2):XII). Los naturalistas ignoraban el aspecto oracional y discursivo de cada lengua, porque percibían las lenguas como lo que les interesaba: un entramado de nombres para enunciar un mundo ya ordenado: Dieu, Soleil, Lune, Étoiles, Terre, Homme, Femme (Saint-Hilaire 1847 (2):108).

Con todo, estos materiales deben apreciarse ante el fondo de lo que en 1813 Johann Severin Vater, basándose en Lorenzo Hervás (1784:26-29), refirió de "51 tribus del Brasil que hablan otros idiomas que el de los tupíes" (Adelung/Vater 1806-17 (III/2):461-466), además del etnónimo mismo: nada. La documentación de algunas de las lenguas ignotas de esos grupos, aunque sea por series de palabras aisladas, parecía altamente deseable. A pesar de esto, Humboldt enfatiza en su respuesta a Olfers las "dificultades de elicitar palabras de individuos de naciones salvajes" y en particular la dificultad de "identificar el significado propio de las palabras alcanzadas de tal manera" (W. v. Humboldt a Olfers 27.6.1821:1). Si no se dispone de buenos diccionarios, que "proceden del conocimiento de la lengua entera" ${ }^{15}$ y que por tanto permiten llegar a los valores

\footnotetext{
${ }^{14}$ El peor caso parece representado por Pohl (1832-37 (1):406), quien declara que según su parecer la lengua de los Kayapó “consiste mayormente de palabras aisladas repetidas varias veces"; lo cierto es que Pohl ha confundido la lista de palabras con la propia lengua.

${ }^{15}$ La entereza propia alcanzable en cada lengua como correlato objetivo de la "impresión
} 
semánticos intrínsecos de esta, a Humboldt le parece "tanto meritoria como útil" la colección aun de palabras aisladas (ibid.). Sin embargo, le concede a Olfers un elogio particular porque este ha comparado unas listas de palabras entre sí, un buen inicio de trabajo filológico: "Vuestra Excelencia adquirirá méritos grandísimos no solamente de mí, sino de la lingüística, si continúa dedicando su cuidado a este objeto y favoreciéndome con comunicaciones ulteriores" (p. 2).

\section{Las demás cartas}

En su segunda carta conservada, del 6 de noviembre de 1821, Wilhelm von Humboldt se propone usufructuar de la nueva posición de Olfers en Lisboa que, entretanto había sido nombrado encargado de negocios; le ruega que busque allí gramáticas y diccionarios de lenguas americanas, tanto en las tiendas de antigüedades, para comprarlos para él, como en la "biblioteca real", ${ }^{16}$ para mandar copias. Una lista de los libros sobre lenguas americanas que Humboldt ya posee, escrita por Louis Friedrich Sachse, escribano de Wilhelm von Humboldt y litógrafo (cf. Schlagenhauff 2000), está anexada a esta carta (W. v. Humboldt a Olfers 6.11.1821, fol. 2r). Olfers responde en carta del 26 de enero de 1822, que Humboldt incluyó en sus papeles de lingüística americana (en el tomo Coll. ling. fol. 52), porque Olfers lo informaba ahí sobre los libros de lenguas que se habian hallado en las bibliotecas. ${ }^{17}$ La carta a Humboldt del 12 de marzo de 1822 ofrece el mismo tipo de informaciones, discutiendo los catecismos en lengua kiriri de Mamiani (1698) y Bernardo de Nantes (1719) y dando una reseña de los diccionarios manuscritos del tupí que se conocían (Olfers a W. v. Humboldt 12.3.1822, fol. 142v).

Después de un período como consejero de la legación en Nápoles, entonces capital del Reino de las dos Sicilias, en septiembre de 1826 Olfers fue destinado a desempeñar la función de encargado prusiano de negocios en Rio de Janeiro, en ese momento ya capital del Brasil independente (Rave 1962:109). La carta que le escribió Wilhelm von Humboldt el 26 de julio de 1826 llegó a manos de Olfers cuando este ya preparaba su partida para Brasil. Esta carta tenía dos anexos: una lista - redactada en francés - de los libros sobre lenguas americanas

total" está al inicio de la lingüística humboldtiana de las lenguas (Humboldt 1905:1) y lo distingue de los meros coleccionadores de lenguas como Murr, quien al fin dijo con resignación: "Immensum Linguarum campum, quantum licuit viribus meis, peragrare tentaui" (Murr 1804:3).

${ }^{16}$ Así llama Humboldt la Biblioteca pública de Lisboa, según el nombre de la biblioteca berlinesa.

${ }^{17}$ Humboldt no reaccionó al catecismo tupí de Araujo (1618), porque no tenía confianza en el modo cómo los "misioneros" trataban la estructura propia de sus lenguas. En cambio, de la gramática Kiriri de Mamiani (1699) hizo hacer una copia (Coll. ling. qu. 49), pero renunció a hacer copiar la gramática de Huerta (1616), sin duda porque las gramáticas del quechua que ya tenía le parecían suficientes. 
que Humboldt quería que se buscasen para él (Olfers la hizo litografiar y añadió un ejemplar a su carta de respuesta del 27 de octubre de 1827), y una lista redactada en alemán - destinada al propio Olfers, con preguntas que se referían a la fonética de varias lenguas americanas. Humboldt percibe una diferencia de accesibilidad entre fonética y gramática al observar una lengua extraña:

Es mucho más importante tener dos o tres lenguas americanas tratadas meticulosamente así como la griega o el sanscrito, en vez de un montón de anotaciones aisladas de más de 60 o 70 de esas lenguas. En lo que concierne a la estructura interior, los viajeros apenas pueden hacer más que llevar consigo unas notas que antes han sido reunidas en el país. Penetrar de verdad en una lengua es una cuestión de muchos años de trato con los indígenas. Pero en lo que atañe a la pronunciación, es mucho más fácil en poco tiempo entender y anotar lo que es preciso. ${ }^{18}$ (W. v. Humboldt a Olfers, 26. 7. 1826, fol. 175 r)

Habla de su trabajo corriente en una "tabla comparativa de los sonidos de las letras de las lenguas americanas conocidas", donde ya ha aprehendido las lenguas mayores (fol. $175 \mathrm{r}-175 \mathrm{v}$ ) y se refiere a las preguntas sobre la lengua "brasiliana" (el tupí), contenidas en la lista para Olfers. ${ }^{19}$

El 13 de agosto de 1826 Wilhelm von Humboldt escribió otra carta a Olfers, donde le pide actuar como mediador con Gui Thomas Marlière, autor de un diccionario Francés-Coroado que pasó a través de un Marqués de Rougé y de Alexander von Humboldt a las manos de Wilhelm (Coll. ling. fol. 35). Wilhelm, por tanto, entendía la presencia de este manuscrito en su biblioteca

\footnotetext{
${ }^{18}$ En alemán: "Es ist viel wichtiger, zwei oder drei Amerikanische Sprachen ganz genau u. gerade so, wie man Griechisch u. Sanskrit behandelt, bearbeitet, als einzelne Notizen über 60 oder 70 aufgehäuft vor sich zu haben. Ueber den innern grammatischen Bau können Reisende schwerlich mehr, als schon im Lande gesammelte Notizen mitbringen. Es gehört langjähriger Umgang mit den Eingebornen dazu, wirklich in eine Sprache einzudringen. Aber über die Aussprache ist es viel eher möglich, in der Kürze das Richtige aufzufassen u. mitzuschreiben". Humboldt concluye este pequeño programa de filología americana con la idea de verificación de las descripciones fonéticas existentes: "la comparación de observciones exactas de lo que hoy se habla, con las afirmaciones de los misioneros, es altamente instructiva para la crítica, tan importante a quien emplea los escritos de estos últimos" (fol. 175r).

${ }^{19}$ En su gramática guaraní, Humboldt menciona la suposición de Olfers de que la alternancia de los "tonos $p, b, m b$ " (en casos como upe, la posposición del objeto indirecto, e yxebe 'a mí', peême 'a vosotros') pertenecería a la escritura, mientras en la pronunciación estaría un único "sonido intermedio", difícil de pronunciar para los no indios (Humboldt 2011, p. 259). En las notas a la tabla de las letras americanas (in: Humboldt en prensa) Humboldt duda si una lengua puede tener "un solo sonido para aquellas tres designaciones" (nota 153, Coll. ling. fol. 146, cartera 8, fol. 356 r). Olfers evidentemente conoce el tupí solamente por fuentes escritas. La unidad de sonido que imagina es la superposición de la lenición de /p/ en $/ \mathrm{v} / \mathrm{y}$ de la nasalización en $/ \mathrm{mb} / \mathrm{o} / \mathrm{m} /$, una tentativa interesante de morfología auditiva.
} 
como un asunto delicado (W. v. Humboldt a Olfers, 13. 8. 1826, fol. 176 r). Trabajando en la tabla de las letras americanas (Coll. ling. fol. 151, cartera III; cf. W. v. Humboldt en prensa) consultó el diccionario de Marlière (Coll. ling. fol. 35) y pensó "que sería indicado escribirle, para sugerirle que redactase también unas notas gramaticales y para hacerle algunas preguntas en cuanto a la pronunciación" (fol. 176r). En el tomo Coll. ling. fol. 50 ("Notas gramaticales sobre lenguas americanas"), cartera 6 ("Gramática coroata"), existe un borrador sin numeración de páginas, con 15 puntos en lengua francesa, destinado a Marlière (Coll. ling. fol. 50, cartera 6, fol. *4r-*5r); Humboldt discute la ortografía aplicada por Marlière, basándose en las explicaciones dadas por Marlière sobre todo en la "Introduction" del diccionario (Coll. ling. fol. 35, fol. *3v, en la cuenta de Humboldt: p. 2). La mencionada carta a Olfers, del 13 de agosto de 1826, debe haber ido acompañada de una copia en limpio de los 15 puntos (y una carta a Marlière).

La respuesta de Olfers es del 23 de octubre de 1827: ha tenido contacto epistolar con Marlière, cuando este pasó por Ouro Preto, y comunica su respuesta (Marlière a W. v. Humboldt, 9.7.1827). Olfers insiste en que Marlière "sin duda tratará de responder a las preguntas contenidas en la carta y su anexo" y responde, en lugar de Marlière, las 15 preguntas sobre la pronunciación de la lengua coroada ${ }^{20}$ (Olfers a W. v. Humboldt, 23. 10. 1827, fol. 144r; 145v-146v). Además, tiene noticias referentes a libros sobre lenguas americanas y sobre la lista litografiada de los libros que Humboldt quiere adquirir; Olfers asegura que esta lista está circulando también en Argentina, Chile, Perú y Colombia (fol. $145 \mathrm{r})$.

\section{Después de las cartas}

En 1828, Olfers retornó de su puesto diplomático en Brasil. Entre 1832-35 obtuvo un nuevo destino por parte del servicio diplomático, esta vez en Suiza, como ministro plenipotenciario prusiano, pero en seguida cambió de función, dejando el servicio diplomático y entrando al Ministero de la Instrucción Pública (Rave 1962:110). Olfers prosiguió una carrera brillante en Berlín.

Wilhelm von Humboldt, por su parte, en los últimos años de su vida se dedicó a las lenguas polinésicas y malayas y a su proyecto de un libro sobre la lengua Kavi en la isla de Java. Sus colecciones americanas quedaron bajo los cuidados de Eduard Buschmann, su colaborador desde 1829, para una

\footnotetext{
${ }^{20}$ Olfers emplea esta ocasión para desaprobar la forma Coroatos que Humboldt da al nombre de los Coroados; la explica por el modo de hablar de Eschwege, originario de la región de Hesse, que habría confundido /d/ y /t/ (fol. 145v). Es verdad que Eschwege presenta, con "Botecudos" (en vez de "Botocudos") y "Xigriaba" (en vez de "Xicriabá"), muestras del fonetismo de su región, pero no de hipercorrección (como sería /t/ en vez de /d/ regional). Por tanto parece más plausible atribuir la forma Coroatos o Croatos a Marlière y alguna reminiscencia suya personal de los croatas europeos.
} 
publicación ulterior que, no obstante, nunca tuvo lugar durante la larga vida de Buschmann. Así también las listas de palabras procuradas por Olfers (Coll. ling. fol. 45 y eventualmente 150) quedaron sin ser utilizadas por la investigación.

Existe una última carta de Olfers a Buschmann, del 21de diciembre de 1836, para obtener el tomo primero de la edición póstuma de la obra de Wilhelm von Humboldt sobre la lengua Kawi (Humboldt 1836).

El 21 de julio de 1839, Olfers fue nombrado director general de los museos reales en Berlín, a pesar de carecer de experiencia profesional en historia del arte, pero sí contando con el apoyo eficiente de Alexander von Humboldt y con la benevolencia del rey (Rave 1962, S. 112, 114).

Alexander von Humboldt sin duda apreciaba la vastedad de la cultura de Olfers, de amplitud mundial y que trascendía las contradicciones aparentemente inconciliables de las especializaciones. A manera de elogio, Alexander evocó los servicios que Olfers había prestado a su hermano Wilhelm. Los estudios americanos de este estaban marcados, por un lado, por la voluntad de alcanzar una "impresión total" de cada uno de sus objetos de estudio, concretamente, de las lenguas, y por otro lado, por la conciencia clara de hallarse ante "una multitud gigantesca de detalles aparentemente insignificantes" (Humboldt 1905:1), pero que, por supuesto, no lo son. La grandeza del gesto de Olfers consiste en el hecho de haber reconocido la diferencia entre sus propias tareas y las de Wilhelm von Humboldt: Olfers procuró los datos impresos y manuscritos sin arrogarse la capacidad de llevar a cabo una interpretación. En la apreciación de las listas manuscritas, los dos hermanos Humboldt juzgaron diferentemente: para el lingüista Wilhelm quedó un expediente aceptable apenas en una situación en la que (aún) no había documentación mejor sobre la lengua respectiva, en cuanto a su funcionamiento; Alexander, en cambio, que había conocido el uso práctico de las listas de palabras, ${ }^{21}$ las considera más bien en función de su valor documental ulterior.

\section{Bibliografía y referencias documentales}

Cartas

W. v. Humboldt a Olfers, 27. 6. 1821, Berlín, AST, Inv.-Nr. 2054.

W. v. Humboldt a Olfers, 6. 11. 1821, Berlín, Staatsbibliothek PK, Autogr. I/282.

W. v. Humboldt a Olfers, 26. 7. 1826, copia, Universitätsbibliothek Jena, Nachlass Leitzmann, Inv.-Nr. 250.

${ }^{21}$ El propio Alexander había empleado listas de palabras, por ej. (A. v. Humboldt 181425 , tomo 2, p. 366367) una comparación de palabras pareni (yabitero) y maipures, dos lenguas arawak o maipuranas. Las palabras pareni fueron elicitadas por Aimé Bonpland y Alexander von Humbodt de dos informantes (p. 365, nota 4), las de la lengua maipure - tomadas de las obras de Gilij (1780-84) y Hervás (1787), además de algunas elicitadas de un informante (p. 366, nota 1). Cf. Zamponi (2003, p. 4). 
W. v. Humboldt a Olfers, 13. 8. 1826, copia, Universitätsbibliothek Jena, Nachlass Leitzmann, Inv.-Nr. 251.

Marlière a W. v. Humboldt, 9. 7. 1827, Cracovia, Biblioteca Jaguelónica, manuscritos, Coll. ling. fol. 52, fol. 133.

Olfers a Buschmann, 21. 12. 1836, Stadt- und Landesbibliothek Dortmund, Atg Nr. 8772.

Olfers a W. v. Humboldt, 26. 1. 1822, Cracovia, Biblioteca Jaguelónica, manuscritos, Coll. ling. fol. 52, fol. 140-141.

Olfers a W. v. Humboldt, 12. 3. 1822, Cracovia, Biblioteca Jaguelónica, manuscritos, Coll. ling. fol. 52, fol. 142-143.

Olfers a W. v. Humboldt, 23. 10. 1827, Cracovia, Biblioteca Jaguelónica, manuscritos, Coll. ling. fol. 52, fol. 144-148.

Otras fuentes manuscritas

Cracovia, Biblioteca Jaguelónica, manuscritos:

Coll. ling. fol. 31 (Vocabulaire coroato-français).

Coll. ling. fol. 35 (Marlière, Vocabulaire français-coroato).

Coll. ling. fol. 45-48 (Wortverzeichnisse amerikanischer Sprachen).

Coll. ling. fol. 50 (Grammatische Notizen über einzelne amerikanische Sprachen).

Coll. ling. fol. 52 (Briefwechsel über die amerikanischen Sprachen).

Coll. ling. qu. 49 (Mamiani, Grammatica Kiriri).

Museum für Naturkunde, Berlín:

SI Sellow, Friedrich, Tagebücher, Nr. 71.

Staatsbibliothek Preußischer Kulturbesitz, Berlín:

Coll. ling. fol. 146 (cartera I, Vergleichende Betrachtung der amerikanischen Sprachen, cartera VIII, Anmerkungen).

Coll. ling. fol. 150 (Onondago Grammatik).

Coll. ling. fol. 151 (Amerikanische Arbeiten).

Textos impresos

Adelung, Johann Christoph, Johann Severin Vater. 1806-17. Mithridates oder allgemeine Sprachenkunde mit dem Vater Unser als Sprachprobe, 4 tomos. Berlín: Vossische Buchhandlung.

Anónimo. 1795. Diccionario portuguez, e brasiliano, obra necessaria aos ministros do altar, que emprehenderem a conversão de tantos milhares de Almas que ainda se achão dispersos pelos vastos certões do Brasil, sem o lume da Fè, e Baptismo. Primeira parte. Lisboa: Officina Patriarcal.

Araujo, Antonio de. 1681. Catecismo Brasilico da Doutrina Christãa, com o Ceremonial dos Sacramentos, \& mais actos Parochiaes. Lisboa: Miguel Deslandes. 
Bernardo de Nantes. 1719. Katecismo da Doutrina Christãa da Lingua Kariris, acrecentado de varias Praticas doutrinaes, \& moraes, adaptadas ao genio, \& capacidade dos Indios do Brasil. Lisboa: Valentim da Costa Deslandes.

Dixon, R. M. W., Alexandra Y. Aikhenvald. 1999. The Amazonian languages. Cambridge: Cambridge University Press.

Edelweiss, Frederico G. 1969. A negação brasiliana "nitio" - "não". In: id. Estudos tupis e tupi-guaranis. Confrontos e revisões. Rio de Janeiro: Livraria Brasiliana, S. 281-284.

Ehrenreich, Paul. 1894. Die Sprache der Cayapo (Goyaz). In: Zeitschrift für Ethnologie 26:115-137.

Eschwege, Ludwig Wilhelm. 1818. Journal von Brasilien, oder vermischte Nachrichten aus Brasilien, auf wissenschaftlichen Reisen gesammelt. 2 cuadernos. Weimar: Verlag des Landes-Industrie-Comptoirs.

- 1830. Brasilien die Neue Welt in topographischer, geognostischer, bergmännischer, naturhistorischer, politischer und statistischer Hinsicht. Primera parte. Braunschweig: Friedrich Vieweg.

Freyreiss, Georg Wilhelm. 1968. Reisen in Brasilien, edición de Stig Rydén. Stockholm: The ethnographical museum of Sweden.

Gilij, Filippo Salvadore. 1780-84. Saggio di storia Americana o sia storia naturale, civile e sacra de' regni, e delle provincie di terra ferma nell'America meridionale, 4 tomos. Roma: Luigi Perego erede Salvioni.

Hassel, Georg. 1816. Allgemeines Europäisches Staats- und Addreß-Handbuch für das Jahr 1816. Segundo tomo, primera parte, Staats- und Addreß-Handbuch für die Europäischen Staaten außerhalb Teutschland. Jahrgang 1816. Weimar: Verlag des Landes-Industrie-Comptoirs.

Hermannstädter, Anita. 2001. Frühe Ethnographie in Brasilien 1815-1831: Die Sammlung Friedrich Sellow und Ignaz von Olfers. Eine Berlin-Brandenburgische Kooperation. In: Gregor Wolff (edición). Die Berliner und Brandenburger Lateinamerikaforschung in Geschichte und Gegenwart. Personen und Institutionen. Berlín: WVB, pp. 313-328.

. 2002: Brasilien - Land der Zukunft. Naturkundliche Expeditionen 18001831. In: Anita Hermannstädter (redacción). Deutsche am Amazonas Forscher oder Abenteurer? Expeditionen in Brasilien 1800 bis 1914. Münster: Lit; p. 26-43.

Hervás y Panduro, Lorenzo. 1784. Catalogo delle lingue conosciute e notizia della loro affinità, e diversità. Cesena: Gregorio Biasini.

. 1787: Vocabulario poligloto. Cesena: Gregorio Biasini.

Huerta, Alonso de. 1616. Arte de la lengua Quechua general de los Yndios de este Reyno del Piru. Ciudad de los Reyes [Lima]: Francisco del Canto.

Humboldt, Alexander von. 1814-25. Voyage aux Régions equinoxiales du Nouveau Continent. Relation historique, 3 tomos. París: Dufour y otros.

Humboldt, Wilhelm von. 1836. Über die Verschiedenheit des menschlichen Sprachbaues 
und ihren Einfluss auf die geistige Entwickelung des Menschengeschlechts. Berlín: Real Academia de las Ciencias.

1905. Ueber das vergleichende Sprachstudium in Beziehung auf die verschiedenen Epochen der Sprachentwicklung. In: Wilhelm von Humboldts Werke, edición de Albert Leitzmann, tomo 4, Berlín: Behr; pp. 1p34.

1994. Mexicanische Grammatik, edición de Manfred Ringmacher. Paderborn: Schöningh.

. 2009. Mittelamerikanische Grammatiken, edición de Manfred Ringmacher y Ute Tintemann. Paderborn: Schöningh.

. 2010. Schriften zur Anthropologie der Basken, edición de Bernhard Hurch. Paderborn: Schöningh.

. 2011. Südamerikanische Grammatiken, edición de Manfred Ringmacher y Ute Tintemann. Paderborn: Schöningh.

2012. Baskische Wortstudien und Grammatik, edición de Bernhard Hurch. Paderborn: Schöningh.

. 2013. Nordamerikanische Grammatiken, edición de Micaela Verlato. Paderborn: Schöningh.

. En prensa. Einleitende und vergleichende amerikanische Arbeiten, edición de Manfred Ringmacher y Ute Tintemann. Paderborn: Schöningh.

Mamiani, Luiz Vincencio. 1698. Catecismo da Doutrina Christãa na lingua brasilica da nação Kiriri. Lisboa: Miguel Deslandes.

. 1699: Arte de grammatica da lingua da naçam Kiriri. Lisboa: Miguel Deslandes.

Martius, Carl Friedrich Philipp. 1867: Beiträge zur Ethnographie und Sprachenkunde Amerika's zumal Brasiliens. 2 tomos, Leipzig: Friedrich Fleischer.

Mueller-Vollmer, Kurt. 1993. Wilhelm von Humboldts Sprachwissenschaft. Ein kommentiertes Verzeichnis des sprachwissenschaftlichen Nachlasses. Paderborn: Schöningh.

1994: Humboldts linguistisches Beschaffungsprogramm: Logistik und Theorie, in: Zimmermann/Trabant/Mueller-Vollmer, pp. 27-42.

Murr, Christoph Theophil von. 1804. Conspectus bibliothecae glotticae universalis propediem edendae operis quinquagintae annorum. Nuremberg: in bibliopolio Monath-Kussleriano.

Pohl, Johann Emanuel. 1832-37. Reise im Innern von Brasilien. 2 tomos. Viena: Anton Strauss's sel. Witwe.

Rave, Paul Ortwin. 1962. Ignaz von Olfers. In: Westfälische Lebensbilder, tomo 9. Münster: xxx:108-131.

Ringmacher, Manfred. 2012. Wilhelm von Humboldts Beschäftigung mit den amerikanischen Sprachen: Kontexte und Perspektiven. In: Jeanette Sakel, Thomas 
Stolz (edición). Amerindiana. Neue Perspektiven auf die indigenen Sprachen Amerikas. Berlín: Akademie Verlag; pp. 9-33.

Rodrigues, Aryon D. 1999. Macro-Jê. In: Dixon, Aikhenvald, pp. 165-206.

Rodrigues, Aryon D., Luciana Gonçalves Dourado. 1993. Panará: identificação lingüística dos Kren-Akarore com os Cayapó do Sul. In: Anais da $45^{a}$ Reunião anual da Sociedade Brasileira para o Progresso da Ciência (2):505. Recife.

Saint-Hilaire, Auguste de. 1847. Voyage aux sources du Rio de S. Francisco et dans la Province de Goyaz. 2 tomos. París: Arthus Bertrand.

Schlagenhauff, Annette. 2000. Die Kunst zu handeln: Louis Friedrich Sachse Lithograph, Kunstförderer und Kunsthändler in Berlin. Jahrbuch der Berliner Museen 42:259-294.

Schwarcz, Lilia Moritz. 2002. A longa viagem da biblioteca dos reis. Do terremoto de Lisboa à independência do Brasil. São Paulo: Companhia das Letras.

Urban, Ignatz. 1893. Biographische Skizzen, I. Friedrich Sellow (1789-1831). Botanische Jahrbücher für Systematik, Pflanzengeschichte und Pflanzengeographie, edición de Adolf Engler, tomo XVII. Leipzig: Wilhelm Engelmann, pp. 177-198.

Zamponi, Raoul. 2003. Maipure. Múnich: LINCOM Europa.

Zimmermann, Klaus, Jürgen Trabant, Kurt Mueller-Vollmer (edición). 1994: Wilhelm von Humboldt und die amerikanischen Sprachen. Simposio Internacional deç Instituto Iberoamericano, Berlín. Berlín, pp. 24-26. Septiembre 1992. Paderborn: Schöningh.

Data recebimento: 25/10/2014.

Data aceite: 20/11/2014. 